\title{
Análisis de los contenidos científicos presentes en Redes Sociales por futuros profesores de Primaria
}

\author{
Amparo Elisa Benéitez Villamor \\ Universidad Complutense de Madrid, Facultad de Educación, Didáctica de las Ciencias \\ Experimentales, Madrid, España
}

[Recibido el 15 de mayo de 2020, aceptado el 16 de octubre de 2020]

La ciudadanía siente como se van modificando los conocimientos científicos que le permiten responder a las cuestiones científico-técnicas, tanto a nivel personal como social. En este trabajo se presenta cómo nuestros estudiantes, futuros docentes en Educación Primaria, organizados en grupos, analizaron los contenidos científicos presentes en diferentes redes sociales. Los documentos presentados por los alumnos fueron analizados para construir una guía de manejo de las redes sociales y para valorar su posible utilización en el aula.

Palabras clave: formación del profesorado, herramientas metodológicas, alfabetización científica, enseñanza de ciencias en primaria

\section{Analysis of scientific content on social media by primary education students}

The scientific knowledge that allows us to respond to the scientific and technological aspects of our social and personal lives is constantly changing and adapting. This article reports on a study of scientific content on different social media by a class of primary education students. The results of their research was analysed to create a user guide to social media for teachers from the point of view of using social media in the classroom.

Keywords: teacher training; methodological tools; scientific literacy; primary science education.

Para citar el artículo. Benéitez Villamor, A. E. (2021). Análisis de los contenidos científicos presentes en Redes Sociales por futuros profesores de Primaria. Ápice. Revista de Educación Científica, 5(1), 21-31. DOI: https://doi.org/10.17979/arec.2021.5.1.6534

Contacto.ampabene@ucm.es 


\section{Introducción}

La ciencia y la tecnología juegan un papel fundamental en nuestra sociedad, pero, la ciudadanía percibe como los conocimientos básicos que le permiten responder a demandas complejas se van modificando en su día a día, tanto a nivel personal como social (Kolst $\varnothing$ et al., 2006). Este requisito, conocido como alfabetización científica, lleva siendo uno de los objetivos de la educación desde hace décadas (DeBoer, 2000; Hodson, 2003; Feinstein, 2011). Una de las causas de las dificultades que siente el alumno para asimilar la ciencia parece ser la falta de conexión entre los contenidos que se trabajan en las clases y en su día a día. Una solución ampliamente citada en la literatura es contextualizar el aprendizaje, relacionando los contenidos trabajados en clase con escenarios cotidianos y familiares para el alumnado (Perrenoud, 2012; Clegg y Kolodner, 2014).

\section{Uso de las redes sociales por los jóvenes españoles}

En España hay alrededor de 28 millones de usuarios de redes sociales y 2 de cada 5 tienen una edad comprendida entre 13 y 34 años (Hootsuite, 2019). El 92\% de estos jóvenes, afirma usarlas a diario, lo cual supone que más de 10 millones de jóvenes y adolescentes se conectan a este tipo de plataformas cada día, y en ellas invierten una media de una hora y cuarenta minutos. Por otro lado, según un sondeo de opinión sobre jóvenes, ocio y consumo del Instituto de la Juventud (INJUVE, 2015), el uso del ordenador es la actividad de ocio más atractiva para los jóvenes, por delante de hacer deporte, ir a discotecas o ver la televisión. Los medios digitales son, por lo tanto, uno de los elementos más presentes en su día a día y contribuyen a la vez a su desarrollo como individuos y a su autonomía personal como ciudadanos, permitiéndoles articular su compromiso social y cultural (Aguaded, Ferrés i Prats, Cruz, Pérez y Sánchez, 2011; Ferrés i Prats y Piscitelli, 2012).

El uso que los jóvenes hacen de las redes sociales es muy variado. Según un estudio de la asociación española de publicidad, marketing y comunicación digital (IAB Spain, 2018), las actividades más realizadas son enviar mensajes, ver vídeos, escuchar música, adquirir información o publicar contenidos (Figura 1).

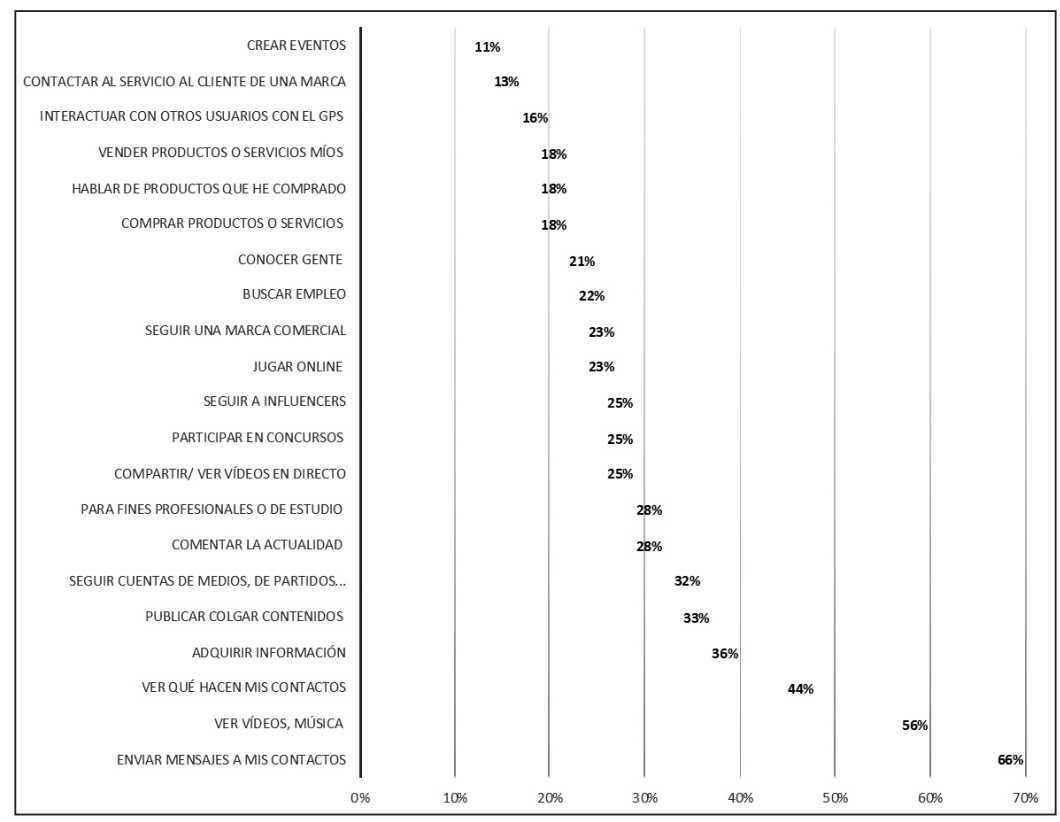

Figura 1. Uso de las redes sociales por parte de los jóvenes españoles según la encuesta de IAB Spain (2018). 
Es de destacar que los menores de 30 años utilizan prioritariamente las redes sociales y los medios digitales para consumir noticias: un $73 \%$ afirma que se informa de la actualidad a través de estas plataformas, lo que contrasta con el $67 \%$ que utiliza la televisión o el $13 \%$ que sigue la prensa (Pardo, 2018). La preferencia por las redes sociales como medio de acceso a la información es consecuencia de las mayores posibilidades que brindan para obtener y transmitir la misma. De hecho, las redes democratizan la disponibilidad de la información y se erigen como uno de los pilares fundamentales sobre los que descansa la sociedad de la información en que vivimos (Castells, 1996, 2001).

Sin embargo, como afirma Fernández Enguita (2011), las redes sociales convierten en "masivo y de coste desdeñable el acceso a la información, pero al propio tiempo, y por ello mismo, vuelven más escaso, más costoso, desigual y jerárquico el conocimiento". Emerge así la obligación de fomentar una aproximación crítica a estas plataformas, una alfabetización mediática vertebrada "en torno a dimensiones compuestas por diversos conocimientos y comportamientos: de lenguaje, de tecnología, de recepción y audiencia, de producción y programación, de ideología y valores, y estética" (Muros, Aragón y Bustos, 2013).

Por otro lado, el conjunto de técnicas y habilidades necesarias para procesar y obtener valor de la información requiere una formación que no todos poseen o pueden adquirir fácilmente (Haywood, 1995; Lenhart, 2003; USDC, 1999). Así pues, el fortalecimiento de las competencias digitales es igualmente imperativo para los alumnos de Primaria. No obstante, la adquisición de habilidades mediáticas y digitales no tiene por qué ser un fin en sí mismo. El uso continuo y cotidiano de las redes sociales por parte de los jóvenes las convierte, potencialmente, en una de las mayores fuentes de información educativa fuera del ámbito académico. Esto justifica explorarlas como recurso en el contexto de la didáctica y, más concretamente, dentro del aula de ciencias (Lorca-Marín, González y Delgado, 2018).

\section{Ciencia en las redes sociales}

Podemos encontrar ciencia en las películas, la televisión, museos o Internet (Ezquerra, 2003; Pro, 2011), en los videojuegos (Lorca-Marín, Vázquez-Bernal y Rosa, 2014), en la publicidad (Ezquerra, Fernández y Magaña, 2015; Jiménez-Liso, 2000), en las noticias (Ezquerra y Pro, 2006; Jiménez-Liso, Hernández-Villalobos y Lapetina, 2010) y también en las redes sociales (Lorca-Marín, González y Delgado, 2018). Estas constituyen, de hecho, la principal vía de aprendizaje informal de contenidos científicos para los escolares en nuestro país. Así, a pesar de que en la encuesta de Percepción Social de la Ciencia y la Tecnología en España (FECYT, 2018), el 75,7\% afirma que la televisión es el medio que más utiliza para informarse sobre este tema, seguido por el $63,4 \%$ que prefiere Internet, prensa digital, redes sociales y otras webs, los datos indican que Internet supera a la televisión entre las personas de 15 a 34 años. Es más, de entre aquellos que afirman utilizar Internet como principal medio de recepción de información sobre ciencia, el 75,7\% lo hacen a través de redes sociales y el $61,9 \%$ mediante vídeos. Estos medios son más utilizados a medida que desciende la edad -entre 15 y 24 años-, mientras que los medios de comunicación digitales especializados son más empleados por personas con formación universitaria. Estos datos nos llevan a justificar la necesidad patente y la obligación ineludible por parte los docentes de conectar los aprendizajes de los contextos informales, como las redes sociales, con aquellos que se imparten en los centros educativos (LorcaMarín, González y Delgado, 2018).

Las redes sociales no sólo influyen en cómo pueden aprender ciencia los escolares, sino que también tienen implicaciones profundas en la manera en que se lleva a cabo la propia labor científica. Por ejemplo, las plataformas profesionales como Linkedln y ResearchGate permiten a científicos de todo el mundo progresar en la carrera académica; 
las publicaciones en Twitter y Facebook facilitan la disponibilidad de las ideas científicas, favoreciendo el avance de los autores, los grupos de investigación, las instituciones y la ciencia en general. Estas herramientas pueden incluso influir en la percepción social de la ciencia (Van Noorden, 2014). En este sentido, ha habido campañas centradas en mejorar la imagen de los investigadores y destruir algunos estereotipos asociados comúnmente con los científicos. Algunos ejemplos incluyen iniciativas para fomentar las vocaciones científicas entre los más jóvenes, con especial énfasis en colectivos con menor representación, como las mujeres (Collins, Shiffman y Rock, 2016; Díaz, 2018).

Si hay algún elemento de la ciencia realmente revolucionado por las redes sociales es la comunicación de esta, que ha sido tradicionalmente dominada por comunicadores profesionales trabajando de forma directa o indirecta para los medios convencionales (Valenti, 1999). Las redes sociales proporcionan una alternativa a la distribución tradicional de contenidos, ya que eliminan las barreras para que los autores alcancen a una gran audiencia (Juhasz, 2009). Además, estas plataformas se construyen mediante una cultura participativa, algo que está ausente de forma patente en los medios tradicionales (Burgess y Green, 2018). Así pues, los usuarios de redes sociales ya no son meros consumidores, sino participantes activos. La comunicación científica no es realizada exclusivamente por informadores profesionales, grupos con intereses comunes, organizaciones e instituciones privadas y públicas, o aficionados a la ciencia (Claussen y col., 2013; Lo, Esser y Gordon, 2010; Nisbet y Scheufele, 2009).

Como ejemplo, en la situación generada por el COVID-19, un elevado número de personas ha compartido, aportado y construido "ciencia" en tiempo record sobre virología. La particular dinámica de las redes sociales ha contribuido a la creación de estos núcleos de conocimiento para responder a las necesidades inmediatas demandadas por la sociedad (Cinelli y col., 2020).

Por otro lado, la naturaleza social de las redes aporta unos elementos diferenciales a la comunicación científica llevada a cabo a través de estas y que están ausentes de cualquier otro medio de comunicación (Lahuerta y Cordero, 2018; Lozares-Colina, 1996; Myers, 1987). La expresión libre de sentimientos y opiniones permite generar vínculos que trascienden la amistad, la familia o el trabajo, y que se basan en el interés por un tema común. De esta forma, los miembros que forman parte de las redes sociales se integran en comunidades virtuales en las que pueden desarrollar su identidad social, lo cual fomenta la lealtad y los comportamientos grupales (Bergami y Bagozzi, 2000; Meyer, Stanley, Herscovitch y Topolnytsky, 2002). Por añadidura, los integrantes de estas comunidades pueden interactuar directamente con los generadores de contenidos, siempre que lo hagan acorde a una serie de normas de grupo, las cuales aceptan voluntariamente al ser coherentes tanto con los objetivos comunes como con sus propios motivos individuales (Postmes, Spears y Lea, 2000).

Según Blanchard (2008), que los miembros de la comunidad se ajusten a estos códigos desemboca en que sus vínculos con el grupo se fortalezcan. En el caso de comunidades de temática científica, estos vínculos son marcadamente activos entre los generadores de contenidos y los demás participantes de la comunidad. En muchas ocasiones los autores, interpelados por los usuarios, pueden adaptar sus contenidos a las demandas de éstos y proponerles preguntas sobre los mismos. Los usuarios a su vez pueden exponer sus respuestas a los demás integrantes de la comunidad mediante materiales de propia factura.

El que un contenido funcione en redes sociales depende fuertemente del formato en que se realice. El texto sigue siendo fundamental, pero los vídeos, fotografías, infografías, etc. son los elementos con mayor aceptación. Según Polinaro (2017), diversos estudios muestran que los contenidos visuales en las redes sociales tienen mayor impacto. Por ejemplo, 
el $75 \%$ de los contenidos publicados o compartidos en Facebook son fotos, las cuales se llevan un $87 \%$ de las interacciones de los seguidores. A su vez, del $63 \%$ de los tuits más reenviados, el $35 \%$ contienen fotos y el $28 \%$ vídeos, mientras que el $82 \%$ de los usuarios afirma consumir contenido audiovisual a través de esta plataforma. Este panorama demuestra que prima el elemento audiovisual, y el vídeo en particular, como canal básico de la comunicación a través de redes sociales, viéndose reflejado en la mayor red social dedicada a la distribución de vídeo en Internet: la página web YouTube (Burgess y Green, 2018; Geipel, 2018). Esto es así tanto en general como en el caso específico de la comunicación científica. Por otro lado, caracterizar el impacto de estos contenidos depende fuertemente de cada red social, pues cada una mide las interacciones de diferente forma.

\section{Diseño de la experiencia}

Con esta idea, se planteó formar a nuestros futuros profesores de Primaria, actuales alumnos de la asignatura obligatoria "Fundamentos y Didáctica de la Biología", del último año del Grado de Maestro de Educación Primaria de la Universidad Complutense de Madrid, para que pudieran detectar e incorporar entre sus competencias la capacidad de conectar lo aprendido en las asignaturas de ciencia con las necesidades sociales de sus futuros alumnos. Esta propuesta inicial, de carácter muy abierto, fue debatida y supuso una importante reflexión sobre el papel del profesorado y los conocimientos de ciencia en el día a día de los ciudadanos. La dinámica que se estableció condujo a los estudiantes a considerar cómo un futuro ciudadano recibirá información científica una vez salga del sistema educativo reglado y cómo podrá seleccionar y contrastar la información que necesite. En concreto, en este trabajo, se presentan las conclusiones elaboradas por nuestros estudiantes, organizados en grupos de trabajo, sobre cómo recoger, analizar y utilizar los contenidos científicos presentes en diferentes redes sociales.

El procedimiento de debate consistió en plantear al conjunto del grupo-clase como cuestión inicial ¿Cómo buscarán información sobre cuestiones científicas vuestros futuros alumnos de Primaria? Esta pregunta inicial generó un amplio conjunto de respuestas que fueron agrupadas en varios apartados: (1) redes sociales: Instagram, Facebook, Twitter...; (2) buscadores: Google, Yahoo...; (3) bibliotecas-videotecas: YouTube, Wikipedia...; y (4) redes cooperativas con el uso de WhatsApp: amistades, expertos, médicos, profesores...

Después de este primer trabajo cooperativo se decidió centrar los esfuerzos en analizar el uso de las redes sociales, dado que esta respuesta obtuvo el consenso de los participantes. Para ello, se estructuró el conjunto de los 15 alumnos participantes en tres grupos. Cada equipo de estudiantes debía analizar, por separado, el uso de las redes sociales en ciencia. En términos pedagógicos, el proceso indagativo para llevar a cabo el proyecto de aula se puede dividir en fases (Rodríguez-Arteche, Bárcena, Rosa y Martínez Aznar, 2019):

- Introducción a la temática y debate del proyecto a realizar.

- Planificación del proyecto y del trabajo en equipos.

- Recogida de la información y análisis.

- Reflexión y elaboración conclusiones.

Las producciones obtenidas por cada grupo se sometieron a un análisis de contenido documental (Cohen, Manion y Morrison, 2013) que utilizó como marco de referencia parcial las categorías obtenidas por Gómez-Aguilar, Roses-Campos \& Farias-Batlle (2012). Este análisis de los documentos de los estudiantes se consideró desde dos puntos de vista. Por una parte, se ordenaron las indicaciones de los estudiantes para construir una guía de manejo de las redes sociales. Por otra parte, se analizaron las afirmaciones desde el punto de vista de la utilización de las redes sociales en el aula. 


\section{Resultados}

Los documentos preparados por los 3 grupos de alumnos fueron inicialmente presentados por cada equipo al resto de sus compañeros. Posteriormente, se planteó un análisis y un primer intento de agrupar las conclusiones. Aquí mostramos los resultados alcanzados por los estudiantes para construir una guía de manejo de la red social y desde el punto de vista de la utilización de las redes sociales en el aula.

\section{Guía de manejo de la red social}

Dado el elevado número de sitios con contenidos de ciencia, los futuros profesores consideraron muy importante enseñar a sus futuros alumnos los distintos tipos de búsqueda que se pueden hacer:

- En Instagram es posible realizar la búsqueda: (1) introduciendo un \#hashtag concreto (por ejemplo: \#ciencia, \#astronomía, \#biología...); (2) usando un hashtag referenciado en alguna de las páginas ya encontradas; y (3) utilizando los enlaces que proponen algunas páginas.

- En YouTube: Los autores pueden utilizar las etiquetas (tags) que son uno de los aspectos que más influyen en la posición que ocupa un vídeo en los resultados de búsqueda, por encima de aspectos como la retención de audiencias que lleva asociado. No obstante, las etiquetas que los usuarios asocian con los vídeos que suben a YouTube no son públicas, por lo tanto, la precisión de las búsquedas depende del buen etiquetado por parte de los autores.

- En Twitter: podemos también encontrar cuentas que publican principalmente videos con contenido científico, pero debemos tener precaución pues aparecen muchas veces cuentas con el mismo nombre. Tal es el éxito de algunas de estas que tiene varias imitaciones. Si ponemos en el buscador de twitter el nombre genérico, aparecen varias cuentas con el mismo nombre (aunque suelen tener muchísimos menos seguidores que las auténticas).

- En Facebook: hay muchas cuentas que teóricamente hablan sobre ciencia; sin embargo, hemos visto que en aquellas que tienen más seguidores muchas de sus publicaciones no hablan realmente sobre ciencia.

Los estudiantes también registraron el modo de identificar las características de las páginas localizadas. Así cómo el procedimiento para obtener el número de páginas con un determinado hastag; el número de usuarios que siguen una página, el número de publicaciones que muestra una página o el número de personas a las que sigue la página en activo. También, la manera de utilizar las métricas e indicadores sobre canales y vídeos que permiten estimar la popularidad de los contenidos científicos a partir de los datos ofrecidos por herramientas de medición de audiencias web. Estas habilidades, específicas para la búsqueda de información, fueron consideradas fundamentales para saber manejar los contenidos propios de la red. En palabras de un estudiante "si no conoces cómo funcionan los hashtags, es como si no supieras usar un diccionario o una calculadora".

Por último, comentaron lo poco riguroso que podían ser algunas páginas frente a otras como las mantenidas por instituciones como FECYT.

\section{Uso de las redes sociales por parte del profesor en el aula}

El uso de las redes sociales por parte del profesor en el aula, y en concreto de Instagram, es un tema muy abierto aun; sin embargo, los futuros maestros llegaron a plantear varios enfoques. Aquí recogemos solo las grandes líneas propuestas: (1) mantenerse al día de las noticias de ciencia (en especial si se siguen instituciones oficiales), (2) identificación de 
posibles errores (de autores particulares), (3) planteamiento de dudas en clase a partir de los contenidos observados, (4) recogida de recursos didácticos (principalmente vídeos y fotografías) y (5) creación de una página de Instagram por parte del grupo-clase bajo la supervisión del profesor.

Respecto a la recogida de contenido didácticos y, teniendo en cuenta el papel preeminente de la imagen para la transmisión de la ciencia, encontramos en YouTube una de las fuentes más interesantes. En la Tabla 1 presentamos los 10 canales de esta red social con más seguidores a nivel mundial de entre las categorías de ciencia y tecnología y de educación. Los datos están ordenados por número de suscriptores al canal, lo que sirve como indicativo del nivel de fidelización generado por los contenidos publicados. Los datos han sido obtenidos de SocialBlade y Tubalytics el 29 de abril de 2019.

Tabla 1. Lista de los 10 canales de YouTube con contenidos científicos más populares a nivel global (fuente: SocialBlade y Tubalytics el 29 de abril de 2019).

\begin{tabular}{|c|c|c|c|c|c|c|}
\hline Canal & Categoría & País & Suscriptores & $\begin{array}{l}\text { № de } \\
\text { vídeos }\end{array}$ & $\begin{array}{c}\text { Visualiz. } \\
\text { totales }\end{array}$ & $\begin{array}{l}\text { Visualiz. } \\
\text { diarias }\end{array}$ \\
\hline Vsauce & $\begin{array}{l}\text { Ciencia y } \\
\text { tecnología }\end{array}$ & $\begin{array}{l}\text { Reino } \\
\text { Unido }\end{array}$ & 14.366 .525 & 378 & 1.591 .618 .364 & 399.263 \\
\hline $\begin{array}{l}\text { Unbox } \\
\text { Therapy }\end{array}$ & $\begin{array}{c}\text { Ciencia y } \\
\text { tecnología }\end{array}$ & Canadá & 14.336 .325 & 1.603 & 2.859 .802 .026 & 2.771 .590 \\
\hline $\begin{array}{l}\text { Technical } \\
\text { Guruji }\end{array}$ & $\begin{array}{c}\text { Ciencia y } \\
\text { tecnología }\end{array}$ & India & 12.583 .197 & 2.263 & 1.174 .183 .660 & 1.646 .410 \\
\hline $\begin{array}{l}\text { Manual do } \\
\text { Mundo }\end{array}$ & $\begin{array}{c}\text { Ciencia y } \\
\text { tecnología }\end{array}$ & Brasil & 12.041 .532 & 1.455 & 2.066.005.294 & 924.167 \\
\hline $\begin{array}{l}\text { The King of } \\
\text { Random }\end{array}$ & Educación & $\begin{array}{l}\text { Estados } \\
\text { Unidos }\end{array}$ & 11.145 .833 & 901 & 2.281.776.971 & 1.435 .890 \\
\hline $\begin{array}{l}\text { Crazy } \\
\text { Russian } \\
\text { Hacker }\end{array}$ & $\begin{array}{c}\text { Ciencia y } \\
\text { tecnología }\end{array}$ & $\begin{array}{l}\text { Estados } \\
\text { Unidos }\end{array}$ & 11.069 .552 & 1.331 & 2.570 .714 .620 & 670.353 \\
\hline $\begin{array}{l}\text { Coisa de } \\
\text { Nerd }\end{array}$ & $\begin{array}{c}\text { Ciencia y } \\
\text { tecnología }\end{array}$ & Brasil & 9.489 .565 & 1.840 & 2.509.479.773 & 1.209 .540 \\
\hline $\begin{array}{l}\text { Primitive } \\
\text { Technology }\end{array}$ & $\begin{array}{c}\text { Ciencia y } \\
\text { tecnología }\end{array}$ & Australia & 9.419 .031 & 44 & 704.201 .231 & 367.240 \\
\hline TED - Ed & Educación & $\begin{array}{l}\text { Estados } \\
\text { Unidos }\end{array}$ & 9.024 .311 & 1.581 & 1.342 .158 .484 & 1.145 .870 \\
\hline $\begin{array}{l}\text { Kurgesagt - } \\
\text { In a } \\
\text { Nutshell }\end{array}$ & Educación & Alemania & 8.714.169 & 97 & 613.809 .476 & 970.343 \\
\hline
\end{tabular}

Una cuestión importante que destacaron los futuros Maestros a la hora usar YouTube es que existen contenidos que no son apropiados para los escolares o que provienen de fuentes cuya fiabilidad desconocemos. Antes de enseñar los vídeos a los estudiantes, hemos de cerciorarnos - viéndolos previamente- de si contienen todo lo que buscamos que los escolares aprendan. Con respecto a la fiabilidad de las fuentes, siempre podemos limitar nuestras búsquedas de contenidos a los canales que ya conozcamos o que pertenezcan a instituciones o corporaciones con una autoridad establecida, lo que ahorra tiempo de búsqueda de entre todos los vídeos. Estos sistemas de limitación de búsqueda son realmente útiles. 


\section{Conclusiones}

La formación de maestros es un tema muy complejo que, entre otras finalidades, implica formar a estos futuros profesionales en el uso de los nuevos recursos. Esta tarea supone, según los propios estudiantes, saber manejar técnicamente estas herramientas y saber adaptar las propuestas educativas a estos nuevos entornos.

Los primeros resultados mostrados aquí indican que es necesario un importante trabajo de ordenación y categorización de las características de estos recursos. En concreto, los docentes en formación consideraron que sus futuros alumnos deberían saber manejar las redes sociales para mantenerse al día de las noticias de ciencia (en especial si se siguen instituciones oficiales), identificar posibles errores (engaños o bulos), y (3) plantearse dudas (consultarlas y/o resolverlas) sobre los contenidos observados. Para lo que estas acciones, que debe hacer el ciudadano -seguir noticias, identificar errores y resolver dudas-, deben trabajarse en clase.

Además, y para la consecución de una correcta formación de los ciudadanos, consensuaron que las redes sociales deberían trabajarse en clase como recurso didáctico (principalmente en formato de vídeo y fotografía) y para crear páginas por parte del grupo-clase bajo la supervisión del profesor.

Obviamente, esto supuso una reflexión sobre la capacidad del docente en cuanto a conocer los recursos existentes (redes sociales: Instagram, Facebook, Twitter...; buscadores: Google, Yahoo...; bibliotecas-videotecas: YouTube, Wikipedia...; y redes cooperativas con el uso de WhatsApp (amistades, expertos, médicos, profesores...). Así mismo, concluyeron que necesitaban alcanzar habilidades específicas para saber buscar y para saber manejar los contenidos en un entorno con sistemas de búsqueda específicos y con modos particulares de categorización o etiquetado (tags, hashtags...).

Tal vez, lo más significativo fue la concreción de estas reflexiones en la necesidad de construir una guía de manejo de redes sociales y en concreto de la utilización de Instagram por parte del profesor en el aula.

En la sociedad actual, los alumnos de Primaria, en su vida cotidiana fuera del aula, están expuestos a interactuar con redes sociales. En este trabajo no pretende incentivar su uso entre los menores, lo que se plantea es mostrar unas pautas de utilización con una ponderada actitud crítica, evitando que se adopten hábitos perjudiciales aprendidos de manera autónoma.

\section{Agradecimientos}

Este trabajo es parte del Proyecto (№ referencia: 39) financiado con el programa Innova Docencia 2018-2019 del Vicerrectorado de Calidad, UCM.

Este artículo es parte del Proyecto I+D+i RTI2018-094303-A-I00: Identificación de Contextos Científicos en la Sociedad. Herramientas para Docentes y Ciudadanos.

\section{Referencias bibliográficas}

Aguaded, I., Ferrés i Prats, J., Cruz, M., Pérez, M. y Sánchez, J. (2011). Informe de investigación: el grado de competencia mediática en la ciudadanía andaluza. Huelva: Grupo Comunicar Ediciones.

Bergami, M. y Bagozzi, R. P. (2000). Self-categorization, affective commitment, and group self-esteem as distinct aspects of social identity in an organization. British Journal of Social Psychology, 39(4), 555-577. DOI: https://doi.org/10.1348/014466600164633 
Blanchard, A. L. (2008). Testing a model of sense of virtual community. Computers in Human Behavior, 24(5), 2107-2123. DOI: https://doi.org/10.1016/j.chb.2007.10.002

Burgess, J. y Green, J. (2018). YouTube: Online video and participatory culture. Malden, MA: John Wiley \& Sons.

Castells, M. (1996). The rise of the network society. Malden, MA: Blackwell.

Castells, M. (2001). La galaxia Internet. Barcelona: Plaza \& Janés Editores.

Cinelli, M., Quattrociocchi, W., Galeazzi, A., Valensise, C. M., Brugnoli, E., Schmidt, A. L., ... \& Scala, A. (2020). The covid-19 social media infodemic. arXiv preprint arXiv:2003.05004.

Claussen, J. E., Cooney, P. B., Defilippi, J. M., Fox, S. G., Glaser, S. M. y Hawkes, E. (2013). Science Communication in a Digital Age: Social Media and the American Fisheries Society. Fisheries, 38, 359-362. DOI: https://doi.org/10.1080/03632415.2013.8162 89

Clegg, T. y Kolodner, J. (2014). Scientizing and Cooking: Helping Middle-School Learners Develop Scientific Dispositions. Science Education, 98(1), 36-63. DOI: https://doi. org/10.1002/sce.21083

Cohen, L., Manion, L. y Morrison, K. (2013). Research methods in education. Routledge.

Collins, K., Shiffman, D. y Rock, J. (2016). How Are Scientists Using Social Media in the Workplace? PLOSONE, 11(10), 1-10. https://doi.org/10.1371/journal.pone.0162680

DeBoer, G. (2000). Scientific literacy: Another look at its historical and contemporary meanings and its relationship to science education reform. Journal of Research in Science Teaching, 37(6), 582-601. DOI: https://doi.org/10.1002/10982736(200008)37:6<582::AID-TEA5>3.0.CO;2-L

Ezquerra, Á. (2003). ¿'Podemos aprender ciencia con la televisión? Educatio Siglo XXI, 20, 117-142.

Ezquerra, Á., Fernández, B. y Magaña, M. (2015). Verdad, mentira... verdad, mentira. Enséñame a decidir. Alambique: Didáctica de las ciencias experimentales, (81), 9-16.

Ezquerra, Á. y Pro, A. (2006). Posibles usos didácticos de los espacios meteorológicos de la televisión. Revista Electrónica de Enseñanza de las Ciencias, 5(1), 114-135.

FECYT. (2018). Percepción social de la Ciencia y la Tecnología en España. Madrid: Fundación Española de Ciencia y Tecnología.

Feinstein, N. (2011). Salvaging science literacy. Science Education, 95(1), 168-185. DOI: https://doi.org/10.1002/sce.20414

Fernández Enguita, M. (2011). Paradojas de la Sociedad de la Información y el Conocimiento. En XI Congreso Español de Sociología. Pamplona: Universidad de Navarra.

Ferrés i Prats, J. y Piscitelli, A. (2012). La competencia mediática: propuesta articulada de dimensiones e indicadores. Comunicar: Revista científica iberoamericana de comunicación y educación, 19(38), 75-82. DOI: https://doi.org/10.3916/C38-2011-02-08

Geipel, A. (2018). Wissenschaft @YouTube. En Knowledge in Action: Neue Formen der Kommunikation in der Wissensgesellschaft. Wiesbaden: Springer VS (pp. 137-163).

Gómez-Aguilar, M., Roses-Campos, S. y Farias-Batlle, P. (2012). El uso académico de las redes sociales en universitarios. Comunicar: Revista Científica de Comunicación y Educación, 19(38), 131-138. DOI: https://doi.org/10.3916/C38-2011-03-04 
Haywood, T. (1995). Info-rich-info-poor: Access and exchange in the global information so- ciety. London: Bowker-Saur.

Hodson, D. (2003). Time for action: Science education for an alternative future. International Journal of Science Education, 25(6), 645-670. DOI: https://doi. org/10.1080/09500690305021

Hootsuite. (2019, 30 de enero). Digital 2019: Global Digital Overview. Recuperado el 12 de mayo de 2019, desde https://www.slideshare.net/DataReportal/digital-2019global-digital-overview-january-2019-v01

IAB Spain. (2018, 5 de junio). Estudio anual de redes sociales 2018. Recuperado el 12 de mayo de 2019, desde https://iabspain.es/estudio/estudio-anual-de-redessociales-2018

INJUVE. (2015). Jóvenes, ocio y consumo. Madrid: Instituto de la Juventud.

Jiménez-Liso, M. R. (2000). Contenidos relacionados con los procesos ácido-base: diagnóstico y propuestas didácticas al nivel universitario (Tesis doctoral, Facultad de Ciencias de la Educación, Universidad de Granada. Granada).

Jiménez-Liso, M. R., Hernández-Villalobos, L. y Lapetina, J. (2010). Dificultades y propuestas para utilizar las noticias científicas de la prensa en el aula de ciencias. Revista Eureka sobre enseñanza y divulgación de las ciencias, 7(1), 107-126.

Juhasz, A. (2009). Learning the five lessons of YouTube: After trying to teach there, I don't believe the hype. Cinema Journal, 48, 145-150.

Kolst $\varnothing$, S., Bungum, B., Arnesen, E., Isnes, A., Kristensen, T., Mathiassen, K., Mestad, I., Quale, A., Vedvik, A. y Ulvik, M. (2006). Science students' critical examination of scientific information related to socioscientific issues. Science Education, 90(4), 632-655. DOI: https://doi.org/10.1002/sce.20133

Lahuerta, E. y Cordero, R. (2018, 23 de marzo). Redes sociales: un antes y un después en el comportamiento humano. Recuperado el 12 de mayo de 2019, desde https:// telos.fundaciontelefonica.com/redes-sociales-un-antes-y-un-despues-en-elcomportamiento-humano

Lenhart, A. (2003). The ever-shifting Internet population: A new look at Internet access and the digital divide. Washington D.C.: Pew Internet \& American Life Project.

Lo, A. S., Esser, M. J. y Gordon, K. E. (2010). YouTube: A gauge of public perception and awareness surrounding epilepsy. Epilepsy \& Behavior, 17, 541-545.

Lorca-Marín, A., González, Y. y Delgado, E. (2018). El uso de las redes sociales en el aula de ciencias: ¿debe ser una obligación o no? En 28 Encuentros de Didáctica de las Ciencias Experimentales. A Coruña: Universidade da Coruña.

Lorca-Marín, A., Vázquez-Bernal, B. y Rosa, S. (2014). Los videojuegos para el profesorado en formación inicial de educación infantil en la enseñanza de las ciencias de la naturaleza. En Investigación y transferencia para una educación en ciencias: Un reto emocionante. XXVI encuentro de didáctica de las ciencias. Huelva: Universidad de Huelva.

Lozares-Colina, C. (1996). La teoría de redes sociales. Papers: Revista de Sociología, (48), 103-126. DOI: http://dx.doi.org/10.5565/rev/papers/v48n0.1814 
Meyer, J. P., Stanley, D. J., Herscovitch, L. y Topolnytsky, L. (2002). Affective, continuance, and normative commitment to the organization: A meta-analysis of antecedents, correlates, and consequences. Journal of Vocational Behavior, (61), 20-52. DOI: https://doi.org/10.1006/jvbe.2001.1842

Muros, B., Aragón, Y. y Bustos, A. (2013). La ocupación del tiempo libre de jóvenes en el uso de videojuegos y redes. Comunicar: Revista científica iberoamericana de comunicación y educación, 20(40), 31-39. DOI: http://dx.doi.org/10.3916/C402013-02-03

Myers, D. G. (1987). Psicología social. Madrid: Editorial Médica Panamerica.

Nisbet, M. C. y Scheufele, D. A. (2009). What's next for science communication? Promising directions and lingering distractions. American Journal of Botany, 96, 1767-1778.

Pardo, P. (2018, 31 de October). Los jóvenes europeos prefieren las redes y la prensa online para informarse. El Mundo. Recuperado el 12 de mayo de 2019, desde https:// www.elmundo.es/television/2018/10/31/5bd8acbae5fdeaa2388b45e2.html

Perrenoud, P. (2012). Cuando la escuela pretende preparar para la vida ¿Desarrollar competencias o enseñar otros saberes? Barcelona: Graó.

Polinaro, J. (2017, 17 de marzo). Redes sociales y ciencia: una pareja que se entiende. Recuperado el 12 de mayo de 2019, desde https://www.madrimasd.org/ informacionidi/analisis/analisis/analisis. .asp ?id $=68513$

Postmes, T., Spears, R. y Lea, M. (2000). The formation of group norms in computermediated communication. Human Communication Research, 26(3), 341-371. DOI: https://doi.org/10.1111/j.1468-2958.2000.tb00761.x

Pro, A. (2011). Conocimiento científico, ciencia escolar y enseñanza de las ciencias de la educación secundaria. En P. Cañal (coord.), Didáctica de la Biología y de la Geología (pp. 13-33). Barcelona: Graó.

Rodríguez-Arteche, I., Bárcena Martín, A. I., Rosa Novalbos, D. y Martínez Aznar, M. M. (2019). Aprendizaje indagativo sobre los cambios físicos y químicos en la formación inicial del profesorado de secundaria. Ápice. Revista de Educación Científica, 3(2), 1-20. DOI: https://doi.org/10.17979/arec.2019.3.2.4657

USDC. (1999). Falling through the net: Defining the digital divide. Washington: U.S. Dept. of Commerce.

Valenti, J. A. M. (1999). Commentary: How well do scientists communicate to media? Science Communication, 21(2), 172-178. DOI: https://doi. org/10.1177/1075547099021002005

Van Noorden, R. (2014, 13 de agosto). Online collaboration: Scientists and the social network. Recuperado el 12 de mayo de 2019, desde https://www.nature.com/news/ online- collaboration-scientists-and-the-social-network-1.15711 
